\title{
Real Time Hybrid Simulation of Electrical Power Systems: Concept, Tools, Field Experience and Smart Grid Challenges
}

\author{
A. Prokhorov ${ }^{\mathrm{a} *}$, Yu. Borovikov ${ }^{\mathrm{b}}$, A. Gusev ${ }^{\mathrm{a}}$ \\ ${ }^{a}$ R\&D Laboratory for Electrical Power Systems Simulation,Tomsk Polytechnic University, 30 Lenin ave, Tomsk 634050, Russia \\ ${ }^{b}$ Electrical Power Systems Department,Tomsk Polytechnic University, 30 Lenin ave, Tomsk 634050, Russia
}

\begin{abstract}
An article discusses achievements in the field of real time hybrid simulation of electrical power systems and their application for smart grid tasks solution. The developed real time hybrid simulation concept and tools are introduced as an alternative to digital simulation systems that are currently widely spread. To substantiate this, the article proposes some factors determining the preference of hybrid simulation approach to numerical methods used in digital simulators. A number of accomplished projects and problems solved in the context of these projects are given to confirm the relevancy, efficiency and viability of the introduced approach of real time hybrid simulation.
\end{abstract}

Keywords: Real time power system simulation, large power systems, intelligent control

\section{Introduction}

Design, research and exploitation of electrical power systems are rather sophisticated issues mostly because of the difficulties of timely receiving of full and reliable data about processes running in them. That is why the simulation is currently a major source of information, the requirements to which are growing with the power systems further complication and emergence of smart grids. The main characteristics of electrical power systems determining requirements to simulation systems are the following: large size, multivariability, stiffness (a ratio of the maximum time constant to the minimum one is $>10^{1}$ ) and also presence of non-linear and dynamic elements. Under such conditions any simplification of the models may cause false conclusions and negative consequences for exploitation in case of the absence of detailed knowledge about behavior of power systems and their processes. On this account a comprehensive tool is required for modeling and simulation of electrical power systems. This tool should provide completeness, adequacy and promptitude of data receiving under the simulation process and should meet the following requirements that must not be mutually exclusive:

- adequacy (accurateness and detail level determining the highest degree of real objects resemblance) of mathematical models of all significant elements that form an electric power system model;

- real time scale and absence of limitations on the duration of simulated processes;

- simulation of entire spectrum of the processes in all possible normal and abnormal regimes of a power system - the use of unified method of mathematical models solution for simulation of quasi-stationary regimes, fast and slow transient processes in a power system of any size without its decomposition and forced simplifications.

The listed requirements constitute some kind of references and thus can be used for evaluation of the state of power system simulation adequacy and time problems as well as for revealing and analyzing factors that interfere with solution of these problems, or for comparison of various approaches.

\footnotetext{
* Manuscript received June 16, 2012; revised August 10, 2012.

Corresponding author: Tel.: +7-906-947-4169; fax: +7-382-2-563-787; E-mail address: antonprokhorov@tpu.ru.
} 


\subsection{Features of numerical methods application}

Impartial assessment of properties and capabilities of numerical methods for power system simulation may consists of the comparison of the given above reference requirements to power system simulators with the characteristics of currently used and widely spread simulation tools utilizing numerical methods.

The analysis of characteristics of these simulators and mathematical models used in them are presented in various publications [1-3] allowed making the following conclusions:

1. Numerical methods of simulation provide successful and effective solution of the static tasks as well as possibility for further development of software tools of this specialization which comes along with advancement of hardware [4]-[6].

2. In case of transient processes simulation, the situation weakly depends on a current level of development of methodological and instrumental base of digital simulation, thus artificial specialization of power systems simulators remains unchanged. This specialization is characterized by evident and constantly existing necessity of deep decomposition of continuous, non-linearly and mutually dependent three-phase processes in power systems [7],[8].

3. The listed difficulties and complications of transient processes simulation cause the necessity of implementation of algebraic and frequency methods for power system dynamic properties evaluation, and essentially steady-state oscillatory stability. Application of these methods does not provide full data about running processes, but still permits the evaluation of dynamic state of given regimes and influence on it by various factors by means of equivalent linearized mathematical power system model.

The experience of analytical and experimental researches of power system simulation problem performed in various publications [9]-[11] define the methodological part of digital simulation of dynamic regimes as the main reason of this problem.

Numerous scientific investigations of differential equation numerical integration methods [11] confirm a heuristic prediction that in the common case a satisfying solution of differential equations by numerical methods is impossible and is only possible for some particular cases under idealized and limiting conditions such as: zero-stability condition which is necessary for convergence of discrete methods and applies some mandatory limitations on the roots of characteristic polynomials, length of solution interval (Dahlquist theorem), the use of implicit methods only and degree of multistep discretization technique which is limited with values $k \leq 2$.

\subsection{Alternative ways of electrical power system simulators evolution}

One of the reasons of intensive development of the mostly spread today digital method of electrical power system simulation were the instrumental errors of analog computers which had been used for a long time for solution of differential equations.

However, while most of the scientists and engineers have been fascinated with the application of the achievements of rapidly progressing digital technologies the idea of application of analog devices for power system simulation has also been developed along with advancement of analog components characteristics. This process has been accompanied by appearance of analog and after those hybrid digital-analog power system simulators.

The main purpose of hybrid simulators development was the reduction of simulation time of power system processes and avoidance of limitations on a power system model size.

But only some prototypes of hybrid simulators capable to simulate quite small power systems have been introduced, besides most of them used analog part for solution of differential equations of generators and their automation systems only while mathematical models of transmission circuit have been performed as algebraic equations solved by digital computers.

Modern analog systems based on operational amplifiers and used for integration of differential equations have much higher accuracy in comparison with systems existed at the time of analog computers prosperity. Though the problem of integrator drift still exists, zero drift is significantly minimized and integration error can be stabilized so well that they influence on the integration results can be neglected. Along with this, the main advantages of analog devices such as real time, methodological error absence 
and absence of limits on a power system model size and simulated processes duration remain.

Nevertheless, analysis of publications shows that concepts of the hybrid simulator stay the same; the only difference is a software and hardware basis $[9,12]$ which continuously improves, but despite this there is no progress in application of theoretical potential of such systems for real exploitation tasks.

\subsection{Requirements to simulation tools and new challenges of smart grids}

Shaping directions of the smart grid development impose new requirements for power system simulators. Along with this a significance of large power systems simulation grows and higher requirements to simulation time are set by the industry. Besides, the deeper integration of IT technologies into power engineering takes place the large number of problems arises, demanding integration of power system simulators into industrial networks and guarantee of its real time operation in continuous interaction with information management systems of power system facilities.

Moreover the development of smart grid technologies implies putting into operation new types of power equipment. For instance, the introduction of FACTS controllers which variety is rapidly growing contributes to a continuous updating of power equipment models database and solution of new level power system control tasks.

In this case resources spent on solution of FACTS models in real time by means of digital simulators are much higher than for traditional types of power equipment. Furthermore, a power system model gets more sophisticated because with implementation of FACTS models the stiffness of differential equation systems also increases. Therefore, the tasks of installation of such complex systems as Generalized FACTS Controllers and analysis of their influence on large power systems, which are actively discussed today, are very unconventional and examples of their full and successful solution are still unknown.

The real time close loop testing of control, protection and automation equipment which are currently solved by digital simulators, are getting more sophisticated in a view of increasing number of information transmitted in smart grid and number of input/output (I/O) signals required. In this regard in digital complexes stability of model solution and real time taken to get a solution depend on a number of I/O signals. At this rate, for instance, problems demanding large power systems simulation and extensive data exchange include design, setting and testing problems of wide area monitoring, protection and control systems (WAMPACS).

\section{Hybrid Approach to Power System Simulation}

It is obvious that technical solutions connected with power systems hybrid simulators development are much more sophisticated and cost intensive. There is no doubt that most suitable solution nowadays is to use considerably improved efficiency of digital systems. However persistent solution difficulties of described problem of power system simulation only with numerical methods and growing with the development of smart grid requirements to power system simulators stimulate a search for alternative methods of simulation.

The guarantee of success in a given search would be application of the approach, that in contrast with digital simulation where the problem is being adopted for tool's functionality, synthesizes a tool to meet the given problems demands. This kind of approach is a hybrid simulation.

\subsection{The concept}

A hybrid simulation technology developed in Tomsk Polytechnic University is based on three simulation approaches: analog, digital and physical. Each approach is used for solution of separate subtasks that form a general power system simulation problem.

A major task of avoiding a methodological error of differential equations solution is handled by continuous implicit integration provided by analog part of simulator, with digital part providing flexible, rapid and accurate model parameters management and results visualization; the existence of physical level makes possible to interconnect the models of power system elements by converting mathematical values into physical model voltages, minimizing with this an amount of digital information exchange. 
Besides, the use of analog switches for commutation of modeled power system elements allows increasing the adequacy of commutation equipment simulation and commutation processes in general. Thereby, the total simulation error is instrumental only and depends on the quality of the hardware and thus minimized with the use of precise components. For instance, the accuracy of modern operational amplifiers and voltage-to-current converters in combination with the use of the methods of offset voltage correction guarantee the maximum error within the full range of performance (with taking into account thermal drift of integrator and instrumental error caused by the analog ground problem) not higher than $0.5 \%$. It should be noted that this error is strictly instrumental and is not accumulated; therefore it does not depend on the size of modeled scheme and duration of real time simulation.

On the basis of the described technology a Hybrid Real Time Simulator of power systems (HRTSim) is built; it represents multiprocessor software hardware system that provides real time continuous implicit integration of differential equations system describing processes in three phase power system. HRTSim allow avoiding decomposition of power system processes and simplification of power equipment models independently on the size of modeled power system. Besides, the capability to provide any longitudinal and transversal commutations makes possible to simulate nay abnormal regime of power system.

\subsection{Hardware part of HRTSim}

HRTSim is based on the modern integrated microelectronics, microprocessor and IT technologies, see Fig.1, a, and have the following structure:

1) It is parallel, multiprocessor, hybrid software-hardware real time system combining adaptable complex of three-phase specialized hybrid processors (SHP) of all simulated power system elements and data management system (DMS);

2) All SHP are built on a common principles; according to these principles, each unit contains coprocessor $(\mathrm{CP})$, corresponding to the particular modeled element of power equipment which system of differential equations it solves, and unified periphery, which includes software-hardware interface of local computer network and longitudinal and transversal digitally controlled three phase groups of single phase commutators;

3) All CP of SHP are also built in a common principles according to which every of them performs microprocessor controlled parallel digital-analog structure providing:

- Continuous and methodologically accurate real time solution of differential equations of certain element: generating unit, power transformer, power line and etc.;

- Conversion: digital-to-analog, analog-to-digital, mathematical analog-three phase physical;

- Automatic and automated control of parameters, settings, longitudinal and transversal phase commutations and etc.;

- Simulation of relay protection, automation and control systems of modeled power equipment.

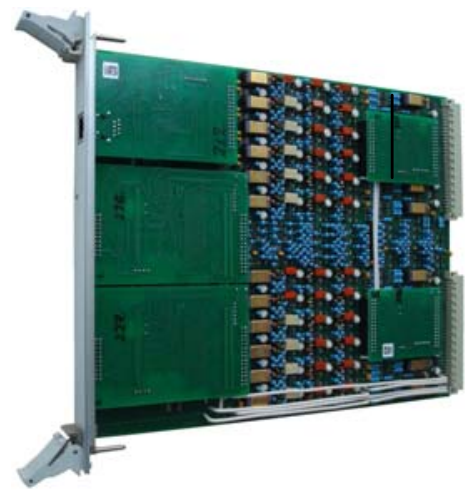

(a)

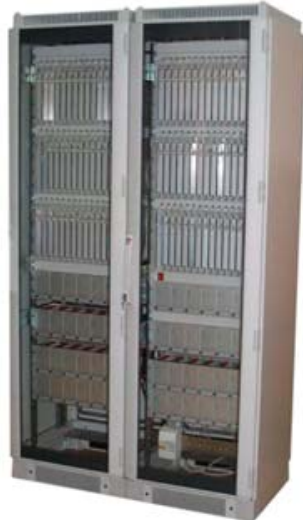
Fig. 1. (a) SHP of power line; (b) HRTSim and its operational diagram: SHP - specialized hybrid processors; LAN - local area
network; TPS - three-phase switch; HWSWI - hardware/software interface; ED - external device; ABC - three-phase input/output of SHP. 
4) Three-phase inputs and outputs of various SHP are connected to each other via three-phase switch in accordance with power system simulation scheme; microprocessor data exchange for all SHP with server computer is performed by means of local area network;

5) HRTSim involves an opportunity of analog signals input and output in a digital form via analog-todigital converter (ADC) of SHP. For interaction with external devices or systems the additional digital-toanalog convertors and amplifiers or protocol translators are provided. On the contrary to the digital simulators, the input/output process and number of analog signals do not influence the time and accuracy of simulation and server load factor, and the ADC performance allows real time exchange of analog signals digitized with $10 \mathrm{kHz}$ frequency.

Input and output $(\mathrm{I} / \mathrm{O})$ of discrete signals are provided by means of discrete $\mathrm{I} / \mathrm{O}$ cards via server without decreasing of system productivity and with maximum time delay of $3 \mathrm{~ms}$.

Listed characteristics in combination with the absence of limitations on modeled power system size and duration of simulated processes make HRTSim a perfect tool for development, setting up and testing of large power systems automation including WAMPACS.

HRTSim architecture also provides significant advantages for FACTS simulation. The accuracy of operating time (for opening and closing) provided by HRTSim is defined with microcontroller frequency (by accurateness of control pulse) and switch opening time (delay on switch tripping and transfer time from one state to another). Frequency of microcontroller managing switches in HRTSim by parallel bus is $120 \mathrm{MHz}$, at the same moment, the time required for internal interruption processing and microcontroller outputs state changing equals to 50-100 ns. Microcontroller outputs are connected to switches inputs driven by logic signals. As the control signal arrives to switch input, the last gets closed within 130-140 ns and gets opened within $100-300 \mathrm{~ns}$ for temperature $0-70{ }^{\circ} \mathrm{C}$.

The accuracy of switch operating time can be evaluated by finding the difference between maximum and minimum time, because the minimum time is set into the controller program. Hence, assuming that operational temperature range inside HRTSim equals $32-40{ }^{\circ} \mathrm{C}$ for external atmosphere temperature 25 ${ }^{\circ} \mathrm{C}$ the maximal operational time error reaches $250 \mathrm{~ns}$.

Considering that amplitude-frequency response of modern microelectronic elements affords frequency range of up to $10 \mathrm{kHz}$ and higher without bringing significant error, HRTSim can perform FACTS controllers based on all existing convertor schemes. In addition SHP building-up principle for FACTS controller just barely differs from SHP building-up principle for traditional equipment. Thus simulation of electrical power systems containing FACTS controllers does not employ increased number of HRTSim software and hardware resources and does not impose any constrains in comparison with traditional power systems simulation tasks.

\subsection{Software part of HRTSim}

HRTSim software is built hierarchically and the following levels are distinguished within its structure:

- Server which provides the liaison with the clients, control of hardware modules, implement regimes and dynamics scenarios, and also runs protection and automation algorithms requiring several modules to be interconnected. The special HRTSim software package is installed at the server machine; the server software contains editable data base, various software tools and provides all data management functions of simulator.

- Client provides interactive interconnection with users, displays modeled power system diagrams which can be manipulated in real time, visualize parameters and characteristics of modeled equipment, performs oscilloscopes and visualizations of control functions, allows user to create scenarios and sends them to the server.

- Specialized client grants interface for control algorithms at the level of connected dynamic libraries (executable files) for the purposes of data collection, data management and formation of external control signals for HRTSim. Specialized client also realizes bidirectional interconnection with SCADA systems by means of standardized industrial protocols.

- Central processor. Every simulation module has central and periphery processors. Central processor interacts with server, governs periphery processors and some module parameters, implement local 
scenarios and functions of upper level of local relay protection systems.

- Periphery processor. There are several types of periphery processors - ADC processors, commutation processors, DQ processors. ADC processor receives analog signals (current, voltage signals and etc.) from analog components of a module and transforms them into digital codes, governs some module parameters, accomplishes calculation of dependable parameters (power flows and etc.), implements starting elements of local relay protection devices. Commutation processor controls circuit breakers and fault-throwing switches. DQ processor transforms signals from DQ to ABC and vice versa.

HRTSim software architecture allows both manual and automatic control of the parameters of the power system model in real time. This becomes possible due to the optimal management of the resources of the simulator information system. Besides there is a capability of increasing HRTSim functionality by adding new additional elements both on the software and hardware level which is especially actual in the context of research and development tasks of the intensively expanding field of smart grid.

\section{Examples of Implemented Real Time Hybrid Simulators And Tasks Solved With Them}

Mentioned above idea of simulator integration into the substations and power stations secondary circuits or automatic dispatching systems for the purposes of solution of a wide range of exploitation tasks, including equipment and network operation regime analysis, automation system setting up and testing is not just a concept, but partially implemented solution based on HRTSim. Besides the problem was defined much earlier than the term 'smart grid' appeared. The first project has been implemented in early $90^{\text {th }}$ and the aim of it was to enhance power systems controllability and observability by integrating HRTSim with power system operative-information complex (OIC) and automation.

In course of time the advent of more advanced systems and devices, as well as new information technologies and standards in electric power industry, such as IEC61850, IEEE C37.118-2005 and etc., has made it possible to bring up to date the concept of integration of HRTSim with software and hardware system (SWHWS) of electric power installations.

\subsection{Examples of the tasks initiated the creation of first version of HRTSim}

The first HRTSim installation in power system and its integration with operative-information complex for exploitation problems solution were implemented in 1998 for Tyumen power system, see Fig.2, a. The differential order of Tyumen power system model exceeded 3500 which corresponded to 600 three-phase nodes, power system model contained 31 electrical machines with turbine/drive mechanism and detailed control systems models as well as described with differential equations models of power lines with the mutual induction, transformers and reactors. Tyumen power system model operational regime was automatically conformed to real measured data continuously received from OIC. Recurrent update of power system data made possible the only reliable verification of HTSim model adequacy to the real power system: according to the data about the generated and consumed power, as well as commutation equipment states, received from OIC, the model regime was set automatically; eventually in result of comparison of voltage levels and power flows distribution in the model and in the real power system a model adequacy had been checked. For instance, the fragments of such comparison are given in Table 1.

As it is illustrated in Table 1, simulation results deviated minimally from real power system data and existing deviations are mostly caused by inaccurate raw data about modeled power equipment and inaccuracies of measurements systems along with the lack of OIC data synchronization.

Proved adequacy and accuracy of the power system model enabled the further creation of HRTSim based real time dispatcher training simulator and successful solution of various power system tasks.

In particular, one of those tasks included setting up of the momentary and sustained fast turbine valving control (FTVC) of Surgut GRES-1 and GRES-2 co-generation power plants for providing dynamic and static stability of Tyumen power system.

The source of the problem was that the lack of an appropriate experimental data led to maladjustment of the FTVC system. As a result FTVC system was overshot which caused out-of-step condition of generating units. 
Table 1. Comparison of voltages and active power flows through power transformers of the $500 \mathrm{kV}$ circuit

\begin{tabular}{lllllll}
\hline Object & Usim, kV & Umeas, kV & $\mathrm{dU}, \mathrm{kV}$ & Psim, MW & Pmeas, MW & $\mathrm{dP}, \mathrm{MW}$ \\
\hline Demyanskaya SS & 502.63 & 505 & 2.37 & 257.44 & 256 & -1.44 \\
Magistralnaya SS & 510.68 & 5083 & -2.68 & 303.02 & 300 & -3.02 \\
Surgut PP 1 & 516.43 & 516 & -0.43 & -2.41 & -1.87 & 0.54 \\
Sibirskaya SS & 506.08 & 506 & -0.08 & 218.78 & 214 & -4.78 \\
Belozerskaya SS & 500.33 & 502 & 1.67 & 274.24 & 276 & 1.76 \\
Trachukovskaya SS & 506.46 & 508 & 1.54 & 391.86 & 388 & -3.86 \\
Kholmogorskaya SS & 508.38 & 506 & -2.38 & 495.04 & 500 & 4.96 \\
Kustovaya SS & 499.56 & 500 & 0.44 & 127.55 & 129 & 1.45 \\
Irtysh SS & 500.88 & 501 & 0.12 & -118.52 & -113 & 5.52 \\
Somkinskaya SS & 512.98 & 515 & 2.02 & 708.49 & 712 & 3.51 \\
Tyumen SS & 504.93 & 505 & 0.07 & -113.45 & -113 & 0.45 \\
Nizhnevartovsk PP & 507.23 & 507 & -0.23 & 0 & 0 & 0 \\
Ilkovo SS & 521.03 & 5225 & 0.97 & 327.16 & 325 & -2.16 \\
\hline
\end{tabular}

One of FTVC automation peculiarities is the necessity to obtain the systems settings considering prefault, fault and postfault regime parameters. Taking into account sufficiently wide range of possible regimes there is a necessity of having set points data base, which should be constantly updated according to the current information about power system operations. Besides, considering the data volume and requirements to the accuracy, the set points calculation must be done automatically, and this in fact provides adaptive properties of such type of power system automation.

The researches carried out on the basis of HRTSim allowed to determine FTVC system settings, providing stable and balanced postfault regime after clearing of the most probable abnormal conditions in power system. The existed overregulation during turbine fast valving was minimized and acceptable transient processes decay time was achieved.

However the lack of possibility to implement an adaptive automation system on the basis of available at that time hardware and complications connected with the full integration of HRTSim into power system control complex caused by technical and administrative obstacles had actualized only manual settings of FTVC system in response to HRTSim research results.

The other successfully implemented task was the research of commutation overvoltages in $500 \mathrm{kV}$ circuit of Tyumen power system. The results of this research were used for defining the measures for overvoltages decreasing and equipment operational conditions optimization. Researches included analysis of overvoltages occurring under different conditions: symmetrical and nonsymmetrical commutations of unloaded power lines with consideration on the voltage phase at the moment of commutation; different short circuit conditions and automatic reclosures, including nonsynchronous commutation, asynchronous and synchronous swings regimes.

Among other factors the results of research have shown that the highest amplitudes are more typical for commutation overvoltages, occurring when $500 \mathrm{kV}$ line phase is being switched-off (taking into account commutation moment) single phase (dead short) fault occurrence (unsuccessful single phase reclosure). For instance, Fig. 2, b illustrates oscillogram of such overvoltage.

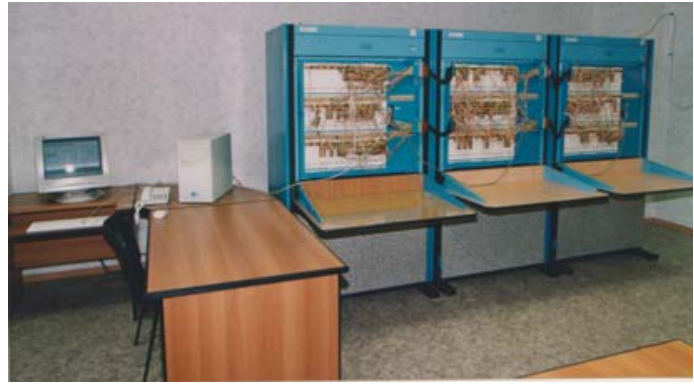

(a)

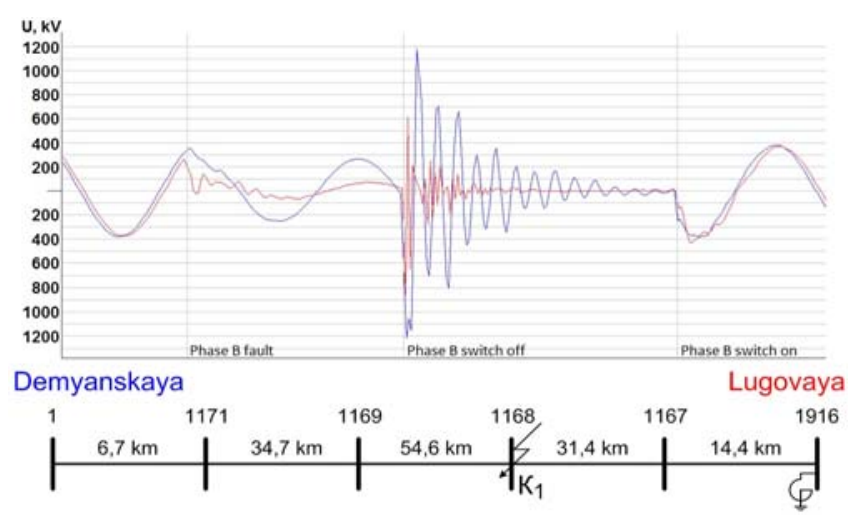

(b)

Fig. 2. (a) First version of HRTSim; (b) Overvoltages after single phase short circuit clearing at two ends of power line. 
On the basis of the first HRTSim version the model of Tomsk power system (Russia) was also implemented and various other researches were done, among them are: setting up and closed loop testing of generators automatic voltage controllers, analysis of synchronous motors self-starting processes for oil and gas company, switching on the parallel operation and synchronization of two power systems.

\subsection{Development of HRTSim for smart grid and large power system simulation tasks}

Implemented in 2008 second version of HRTSim, see Fig.1, is based on the modern information technologies, more advanced hardware and has much more flexible architecture which makes it suitable for vide range of smart grid tasks solution, including the tasks requiring interconnection of HRTSim with external devices and integration with external systems.

Fig. 3 illustrates the simplified functional diagram of HRTSim integration with substation software and hardware system (SWHWS). This diagram shows two ways of integration: first based on IEC 61850 standard is mostly a perspective because the current level of its field implementation allows providing only partial integration; and the second based on the more conventional solutions is implemented within some current projects based on HRTSim.

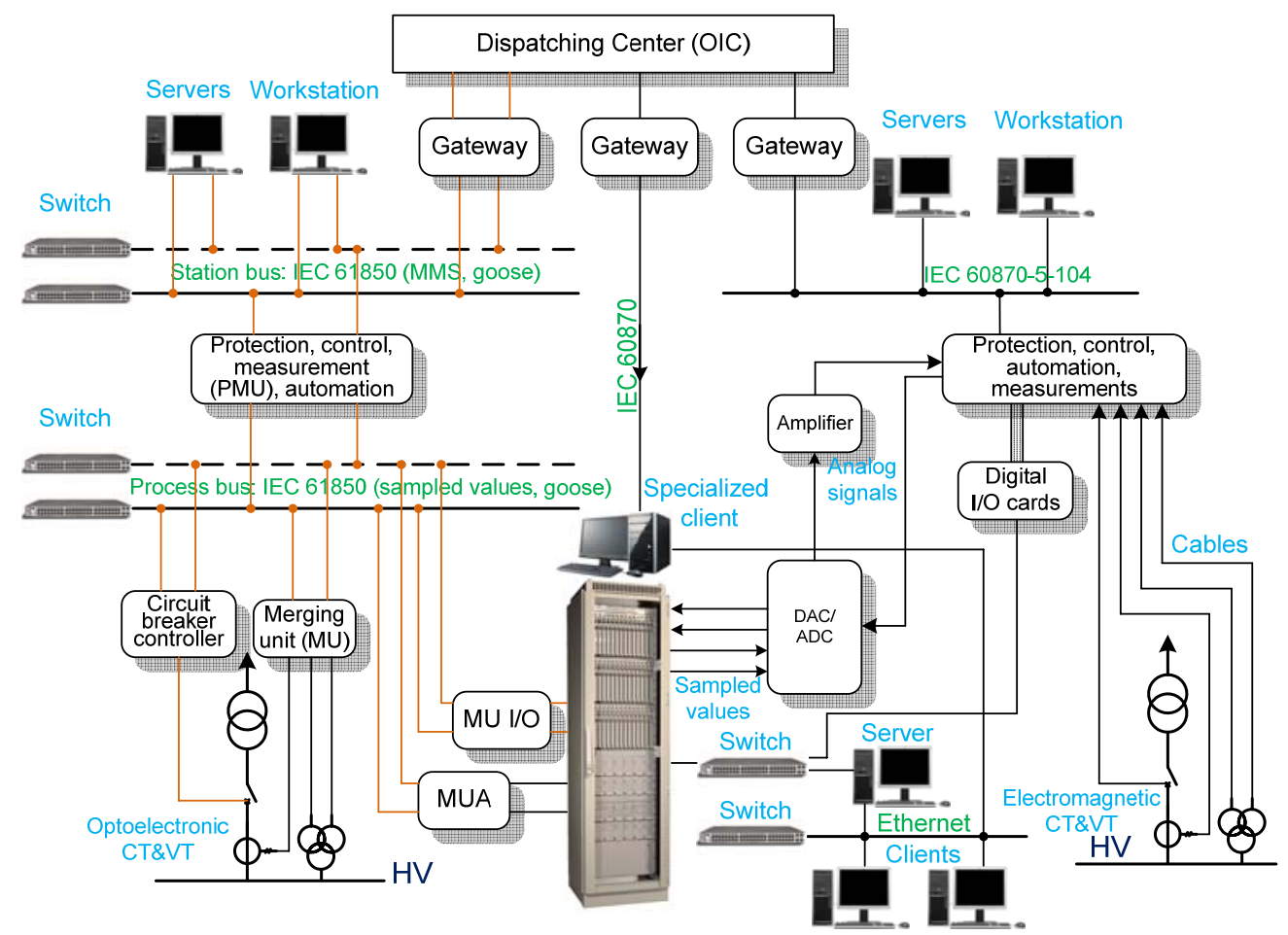

Fig. 3. Functional diagram of HRTSim integration into substation SWHWS.

The approbation of HRTSim new software and hardware base was done within the framework of the project on creation of Tomsk power system simulation complex for JSC Federal Grid Company, Russia (JSC FGC). Installed in Tomsk transmission network utility, HRTSim performs scheme containing 196 three phase nodes, 42 electrical machines and 223 network elements (transformers, power lines, reactors and etc.).

Besides, the other project on large power systems simulation was carried out for JSC FGC on development of $220 \mathrm{kV}$ and higher voltage network simulation scheme of United Power System of Siberia (UPSS) and technical proposal on HRTSim integration into active-adaptive network of UPSS. According to the project the UPSS model will be containing 3000 three-phase nodes, 496 electrical machines, 1752 network elements including controlled shunt reactors, static series compensators, static VAR compensators, HVDC joints.

At this moment the first stage of the project on HRTSim development for Elgaugol energy cluster, which is one of the pilot projects on Russia active-adaptive networks creation, is to be completed. The 
aim of this project is to apply HRTSim for development and testing of adaptive systems for generalized control of a group of substations, and as a part of this to integrate HRTSim with SWHWS of control center of a group of substations of Amursk power system. A scheme of simulated power system including Elgaugol energy cluster and adjacent network is given in Fig. 4.

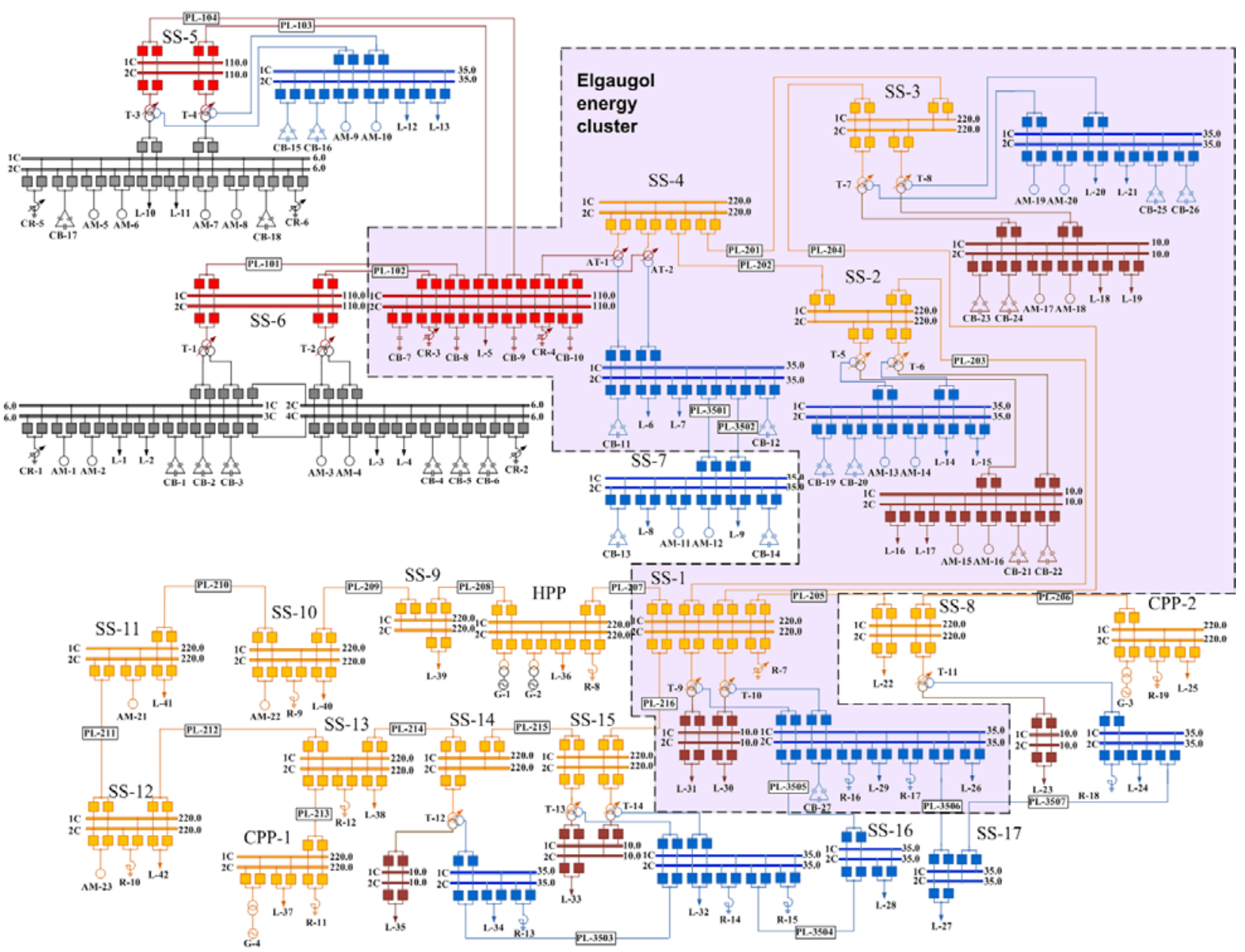

Fig. 4. Simulated power system: SS - substation, CPP - co-generation power plant, HPP - hydro power plant, $\mathrm{PL}$ - power line, $\mathrm{CB}$ - capacitors bank, CR - controlled reactor, $\mathrm{R}$ - switched reactor, L - load, AM - asynchronous motor, $\mathrm{G}$ - generator, (A)T - (auto) transformer.

Particularly the projects solves the problem of testing and setting up of active-adaptive voltage and VAR control system (AAVVCS), intended for generalized control of reactive power compensators (thyristor-controlled reactors and switched capacitors) and automatic tap changer of power transformers of the group of energy cluster substations. The project is divided into three stages:

Stage1. Testing of AAVVCS algorithms at the stage of detail designing.

At this stage the implementation of virtual AAVVCS and its interconnection on program level with HRTSim for data exchange between equipment models, its control systems and AAVVCS model is provided. The analysis including the following tasks should be done during the tests:

- determination of AAVVCS regulator parameters operational range;

- estimation of the sufficiency of prospected VAR compensators installed capacity as well as their numbers and optimality of the installation places;

- assessment of AAVVCS application efficiency;

- analysis of energy cluster and adjacent network operational regime, in particular: examination of steady-state and dynamic stability conditions; research and analysis of normal and abnormal regimes of supply network, energy cluster network and energy cluster consumers including large electrical motors. 


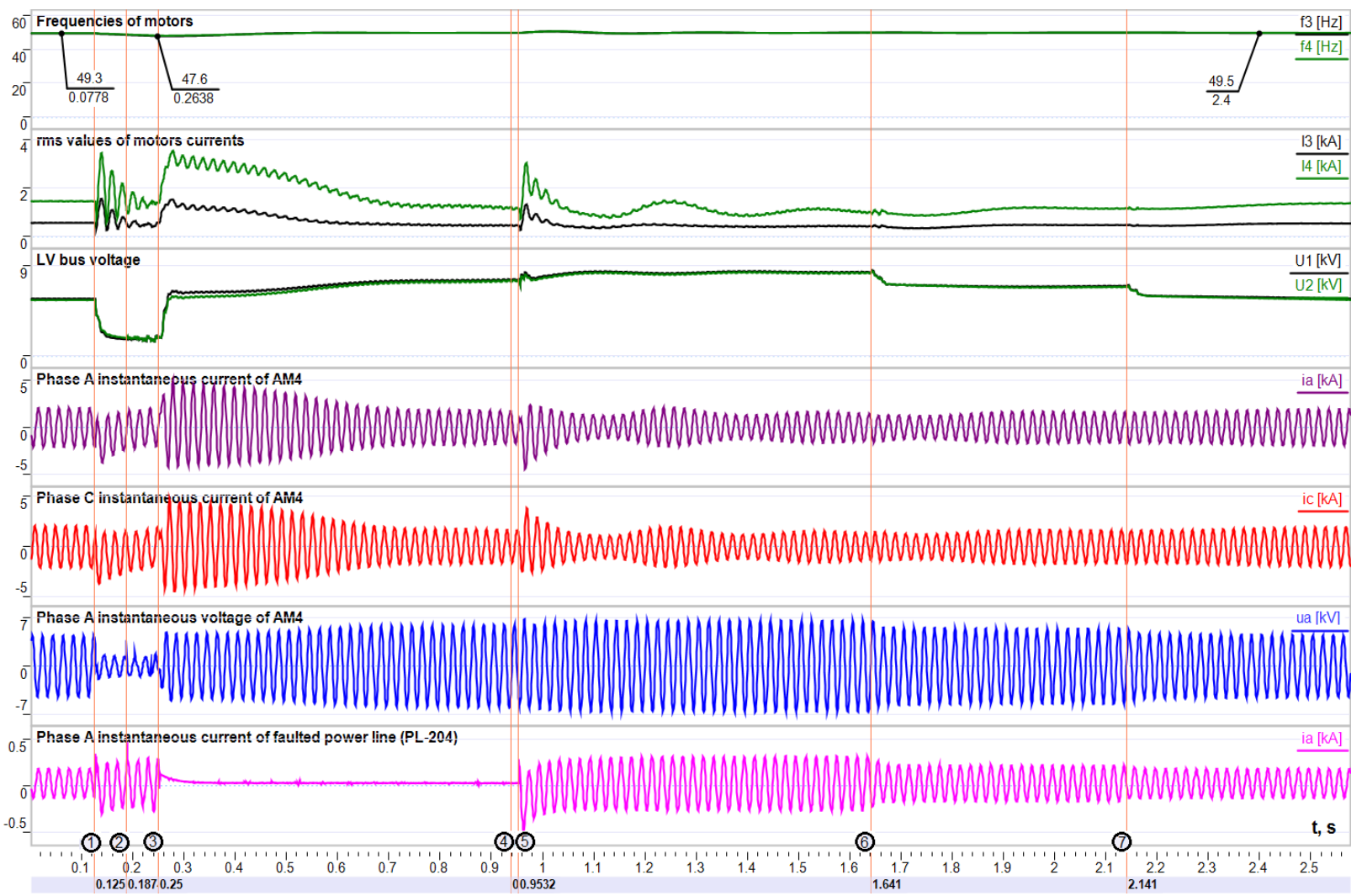

Fig. 5. Results of real time simulation of energy cluster operations.

The example of real time simulation results of self-starting process of energy cluster consumer's asynchronous motors and automation actions in the case of voltage dip occurring when one of the energy cluster supplying power line is faulted is performed in Fig. 5.

At the bottom of Fig. 5 the following main events and automation actions leading to the critical motors successful self-starting are numbered:

1 - three phase fault of power line PL-204 supplying energy cluster;

2 - the following voltage dip causes the forced shedding of thyristor controlled reactors CR-3 and CR4 and sending the capacitors banks CB-1 - CB-4 circuit breakers close commands by their automatic control systems.

3 - power line PL-204 protection trips its circuit breakers off in $0.05 \mathrm{~s}$ after fault occurring;

4 - undervoltage protection of non-critical motors trips them off in $0.5 \mathrm{~s}$ after the power line fault for lightening the self-starting conditions of the motors remaining energized. At the same time auto-reclosing of the power line circuit breakers trips them on.

5 - Successful auto-reclosing lead to further voltage increasing.

6,7 - switching off of the first and then second capacitor bank of each VAR compensator results when the network voltage exceeds the set point of automatic control system of VAR compensator.

Given example illustrates the possibilities of integrated assessment and analysis of power systems operational conditions by means of HRTSim for obtaining individual settings of the generalized control system for each power system regime and thus providing its adaptive functions.

Stage 2. Production testing of AAVVCS of substation level and AAVVCS of substation group control center (SGCC).

In course of production testing a test bench will be developed, the last contains HRTSim as virtual power system counterpart, AAVVCS SS and AAVVCS SGCC prototypes are introduced by hardware and software, configured for energy cluster VAR compensators and tap changers control. The objectives of the given stage which should be achieved by means of HRTSim include:

- AAVVCS regulators quality evaluation; 
- evaluation of the energy cluster stability margins in conditions of AAVVCS operation;

- implementation of production testing program which includes all AAVVCS SS and AAVVCS SGCC functions testing.

At this stage in autonomous regime with the use of preliminary prepared scenarios for various regime situations inherent to energy cluster load and network are simulated.

Scenarios running in real time regime is accompanied with HRTSim and SWHWS of AAVVCS data exchange (telemetering (root-mean-square values of current and voltages, phase-to-ground/phase-tophase), telesignalization of parameters from HRTSim and telecontrol of HRTSim parameters in real time). Data exchange is performed in accordance with IEC 60870-5-104 via Ethernet.

Stage 3. Operational testing.

HRTSim is assumed to be placed at SGCC of energy cluster at the stage of operational testing for automatic setting and following of the energy cluster operational regime according to the telemetering and telesignalization data from real objects. It also employs bidirectional interconnection with energy cluster automation and control equipment, including AAVVCS, which must operate only in alarm regime.

For the purposes of dynamic setting of HRTSim regime in accordance with operational conditions of real power network it is integrated with SWHWS of SGCC for receiving PMU data and teledata from the energy cluster substations. Thus real control objects will be replaced by their dynamic models at the stage of operational testing for the assessment of the correctness and efficiency of AAVVCS and other automation operations. Bidirectional data exchange between HRTSim and SWHWS of SGCC and automation equipment will be arranged in compliance with IEC 60870-5-104.

Thus this project will demonstrate in practice the efficiency of all engineering proposals and suggest new building principals of SWHWS of automated electrical power objects.

\section{Conclusion}

HRTSim is actively developing system, the main advantage of which is its high level of flexibility, allowing continuously increase its functional capabilities without loss of its main features: accuracy, adequacy, real time of simulation, unlimited model expandability and unlimited duration of simulated processes. The above mentioned characteristics of HRTSim provide opportunities of its application for wide range of smart grid tasks, among others are:

- real time dispatcher training and advising;

- IEC 61850 simulator building;

- development, setting up and closed loop testing of protective relay, automation and control equipment;

- real time simulation of large power systems and development of approaches for building of WAMPACS and FACTS with multiple controllers and generalized control system, and also make it possible to consider HRTSim as a part of smart grid in the future.

\section{Acknowledgements}

This paper contains the results of the work supported by State Task «Science» 2.7.2012 "Research and Development of Hybrid Model of Power Plant Generating Units".

\section{References}

[1] CIGRE Task Force 38-02-08. Long term dynamics. Part II: a practical assessment of simulation tools. Summary Report, Convenor: M. Stubbe, Belgium; 1992.

[2] Mohan N, Robbins WP, Undeland TM, Nilssen R, Mo O. Simulation of power electronic and motion control systems-an overview. Procedings of IEEE, 1994; 82(8):1287-1302.

[3] Mahseredjian J, Dinavahi V, Martinez JA. Simulation tools for electromagnetic transients in power systems: overview and challenges. IEEE Transactions on Power Delivery, 2009; 24(3):1657-1668.

[4] Woodruff SL. Complexity in power systems and consequences for real-time computing. Proceedings of the Power Systems Conference and Exposition, 2004; 3:1770-1775. 
[5] Jatskevich J, Wasynczuk O, Mohd Noor N, Walters EA, Lucas CE, Lamm PT. Distributed simulation of electric power systems. In: Proc. of 14th Power Systems Computation Conference, 2002:1-7.

[6] Belanger J, Paquin JN. Real-time simulation of large-scale power systems using EMTP-RV and Simulink/SimPower systems. IEEE Energy Conversion Congress and Exposition, 2009.

[7] Zhang P, Marti JR, Dommel HW. Network partitioning for real-time power system simulation. In: Proc. of the International Conference on Power System Transients (IPST'05), Montreal, 2005: IPST05-177.

[8] Morales F, Rudnick H, Cipriano A. $\varepsilon$ Balanced decomposition for power system simulation on parallel computers. In: Proc. of the 3rd International Meeting on Vector and Parallel Processing, 1998,.

[9] Bond JA, Dorsey JF, Brooke M, Magill J, Tabler J. Fast solution of nonlinear equations using parallel analog hardware. Analog Integrated Circuits and Signals Processing, 1994, 6(2):135-156.

[10] Watson NR, Arrillaga J. Power Systems Electromagnetic Transients Simulation. UK: MPG Books Ltd; 2003.

[11] Hall G, Watt JM. Modern Numerical Methods for Ordinary Differential Equations. USA: Oxford University Press; 1976.

[12] Carullo SP, Olaleye M, Nwankpa CO. VSLI Based analog power system emulator for fast contingency analysis. In: Proc. of the 37th Hawaii International Conference on System Sciences, 2004:1-8. 